\title{
Spectrum Sensing in Opportunity-Heterogeneous Cognitive Sensor Networks: How to Cooperate?
}

\author{
Guoru Ding, Student Member, IEEE, Jinlong Wang, Senior Member, IEEE, Qihui Wu, Senior Member, IEEE,
} Fei Song, and Yingying Chen, Senior Member, IEEE

\begin{abstract}
Cognitive sensor network (CSN) is a promising paradigm to address the spectrum scarcity problem in traditional wireless sensor networks. Reliable spectrum sensing is essential to enable the normal operation of a CSN. Existing researches showed that by exploiting spatial diversity, cooperative sensing can greatly improve the detection performance over noncooperative sensing in opportunity-homogeneous environment. At a given time, cognitive sensors at different locations, however, may experience heterogeneous spectrum opportunities making the cooperation among cognitive sensors intractable. In this paper, we show the limitations and drawbacks of merely using temporal-domain detection performance metrics and introduce novel spatio-temporal detection performance metrics to guide the design of joint spatio-temporal spectrum sensing. An efficient one-bit hard decision based three-phase (i.e., a global cooperation phase, a local cooperation phase, and a joint decision phase) spatio-temporal sensing algorithm is proposed and numerical results demonstrates the effectiveness of the proposed algorithm.
\end{abstract}

Index Terms-Cognitive sensor network, spectrum sensing, heterogeneous opportunity, cooperation.

\section{INTRODUCTION}

$\mathbf{R}$ APID advances in processing capability, memory capacity, and radio technology have enabled the development of wireless sensor networks (WSNs) with small and inexpensive communication sensors [1]. WSNs are capable of monitoring physical and environmental information for various high-level applications. We envision that the wide deployment of WSNs will impact our daily life dramatically. Current WSN sensors usually operate on license-exempt Industrial, Scientific and Medical (ISM) frequency bands, which are shared with many other popular wireless technologies (e.g., Wi-Fi and Bluetooth) and become more and more crowded [2].

To deal with the spectrum scarcity problem, a new sensor networking paradigm, cognitive sensor network (CSN), which

Manuscript received May 12, 2013; accepted May 15, 2013. Date of publication May 20, 2013; date of current version September 25, 2013. This work was supported in part by the National Natural Science Foundation of China under Grant 61172062 and Grant 60932002, the National Basic Research Program of China under Grant 2009CB320400, and by the Jiangsu Province Natural Science Foundation under Grant SBK201122196. The associate editor coordinating the review of this paper and approving it for publication was Prof. Elena Gaura. (Corresponding author: $Q$. Wu.)

G. Ding, J. Wang, Q. Wu, and F. Song are with the College of Communications Engineering, PLA University of Science and Technology, Nanjing 210007 China (e-mail: dingguoru@gmail.com; wj1543@sina.com; wqhtxdk@yahoo.cn; aroucian@163.com).

Y. Chen is with the Electrical and Computer Engineering Department, Stevens Institute of Technology, Hoboken, NJ 07030 USA (e-mail: yingying.chen@stevens.edu).

Color versions of one or more of the figures in this paper are available online at http://ieeexplore.ieee.org.

Digital Object Identifier 10.1109/JSEN.2013.2264161 incorporates cognitive radio [3] capability on the basis of traditional WSNs has been studied recently [4]-[7]. In CSNs, cognitive sensors exploit frequency bands that are licensed to primary users (PUs) but are not used by them at a specific time and/or in a specific area. Since cognitive sensors can operate on licensed frequency bands of the PUs in an opportunistic or interference-free manner, they need to have the capability to identify the availability of spatial and/or temporal access opportunities via spectrum sensing prior to transmission. Thus, spectrum sensing is one of the major functionalities distinguishing CSNs from traditional WSNs. For a frequency band of interest, the problem of spectrum sensing is generally formulated as a binary hypothesis testing: is there an access opportunity or not? The access opportunity can be characterized as temporal or spatial. A temporal access opportunity is a time period when the primary transmission is absent while a spatial access opportunity is a geographical area where the cognitive sensor is far away from the PU [8].

Reliable spectrum sensing is a challenging task due to hidden node problem [9], shadowing effect, multi-path fading and time-varying natures of wireless channels [10]. To address these problems, cooperative sensing (CS) among multiple spatially distributed spectrum sensors is a promising direction [11]-[13], which can achieve much better detection performance than non-cooperative sensing (NCS) by exploiting multiuser diversity [14].

Although the problem of spectrum sensing has been extensively studied, there are still some critical issues needed to be addressed. First of all, a more general system model is needed. Majority of the existing studies (e.g., see [15]-[19]) consider an opportunity-homogeneous CSN, which consists of a large-scale PU network (e.g., a Digital TV system) and a small-scale SU network (e.g., a WLAN-like CSN system). In this kind of network scenario, the distances between any cognitive sensors are very small compared with the distance from any cognitive sensor to the primary transmitter, and thus the average received signal-to-noise ratios (SNRs) at cognitive sensors are approximately equal. Hence, the problem of cooperative sensing in the literature is usually modeled as a common binary hypothesis testing among all cognitive sensors. However, when we consider an opportunity-heterogeneous $C S N$, where the network scales of the primary system (e.g., a Wireless Microphone system) and the cognitive system are comparable, at a given time cognitive sensors at different locations may experience different spectrum opportunities, in which case the common hypothesis does not hold any more. 
Furthermore, improved detection performance metrics are needed. Traditionally, a pure temporal sensing sensitivity is used to evaluate the detection performance of different schemes. For example, in IEEE 802.22 standard [20], for a fixed sensing time, a detection probability no less than 0.9 and a false alarm probability no larger than 0.1 are required when the average received SNR is $1 \mathrm{~dB}$ for analog TVs, $-21 \mathrm{~dB}$ for digital TVs, and $-12 \mathrm{~dB}$ for wireless microphones. This temporal sensing sensitivity is very conservative and usually leads to a over-large spatial margin [8].

In this paper, we study the problem of spectrum sensing in opportunity-heterogeneous CSNs. We introduce a novel network model that can characterize opportunity-heterogeneity among different cognitive sensors and redefine the problem of spectrum sensing in CSNs from a joint spatio-temporal twodimensional detection perspective. We show the limitations and drawbacks when only temporal-domain detection performance metrics are used by reviewing the traditional spectrum sensing schemes (i.e., both NCS and decision fusion-based CS), and derive novel two-dimensional detection performance metrics to guide the design of joint spatio-temporal spectrum sensing. The fact that neither NCS nor CS performs well in opportunity-heterogeneous CSNs motivates us to further propose an efficient three-phase spatio-temporal sensing (TP-STS) algorithm: a global cooperation phase to identify the common temporal opportunity for all cognitive sensors by exploiting multiuser diversity, a local cooperation phase to identify the heterogeneous spatial opportunities for individual cognitive sensors by effectively fusing non-cooperative binary decisions among neighboring sensors, and finally a joint decision phase to determine the availability of the spatio-temporal opportunity at each individual cognitive sensor. Numerical results are provided to demonstrate the effectiveness of the proposed algorithm.

The rest of this paper is organized as follows. Section II describes the system model and problem formulation. Section III presents the performance comparisons of the existing NCS and CS schemes. Section IV presents the technical challenges ahead and develops a novel sensing algorithm. Section V provides numerical results, Section VI reviews the related works, and Section VII concludes this paper.

\section{System Model and Problem Formulation}

We consider a CSN that shares a common frequency band with a primary user system. Of interest in this paper is to determine the availability of a spatial and/or temporal spectrum opportunity for each cognitive sensor in CSN.

\section{A. Opportunity-Heterogeneous Network Model}

As shown in Fig. 1, we consider a CSN with one primary transmitter (PT) and $N$ cognitive sensors distributed in the given geographical area. The PT has a PER with a radius $D_{p}$ [26]. Inside the PER, no cognitive sensor may transmit if the primary transmission is detected, in order to guarantee any potential primary receptions within. Outside the PER, cognitive sensors can share the common frequency band with the PT in a spatial reuse manner.

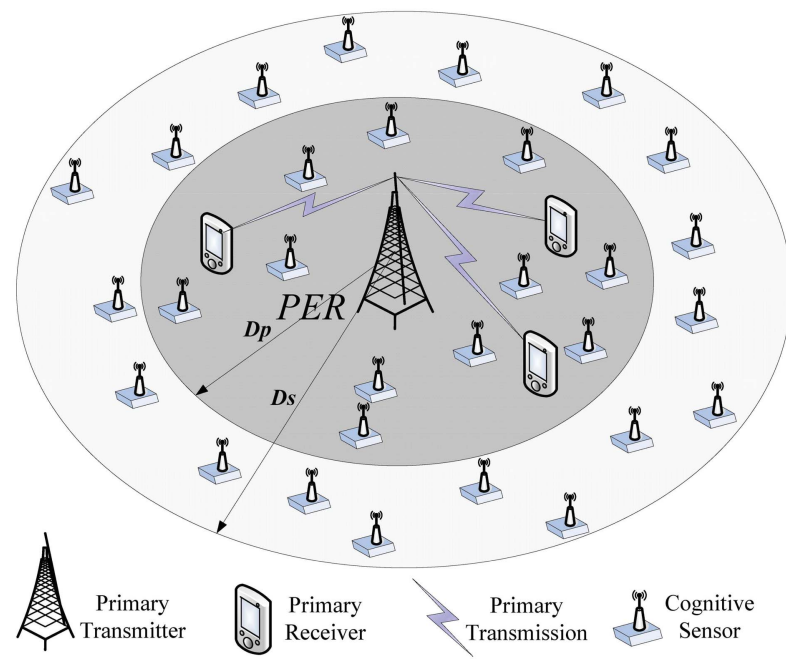

Fig. 1. An opportunity-heterogeneous CSN. $D_{p}$ is the radius of primary exclusive region (PER) and $D_{s}$ is the sensing range of a cognitive sensor.

We further consider that $N$ cognitive sensors randomly distribute in a circular area with a radius $D_{s}$, which is closely related to the sensing sensitivity of cognitive sensors. Any cognitive sensor outside this area will not be able to detect the presence of the primary signal. Typically, $D_{s}>D_{p}$ holds since cognitive sensors must have a higher sensing sensitivity than the primary receiver sensitivity in order to avoid interfering with PRs [27]. In this case, some cognitive sensors can be located inside the PER and only temporal opportunities could be exploited, while others can be located outside the PER and both temporal and spatial opportunities for them are available. Consequently, at a given time different cognitive sensors in CSN will experience heterogeneous spectrum opportunities.

We consider that the cognitive sensors' positions follow a two-dimensional uniform distribution with density $\rho$, i.e., $N=\left\lfloor\rho \pi D_{s}^{2}\right\rfloor{ }^{1}$ Due to the lack of cooperation from the primary user system, we consider that any cognitive sensor $i$ in the CSN does not have the prior location information of the PT and performs opportunity detection based only on the received primary signal strength:

$$
P_{i}\left(d_{i}\right)=P_{t} \cdot \phi_{i}\left(d_{i}\right) \cdot \psi_{i} \cdot \varphi_{i},
$$

where $P_{t}$ is the transmission power of PT, $d_{i}$ is the distance between the PT and cognitive sensor $i, \phi_{i}\left(d_{i}\right)$ is the path-loss component, $\psi_{i}$ and $\varphi_{i}$ are respectively the shadowing fading and multi-path fading components [28].

\section{B. Spatial and Temporal Opportunity Model}

This section formulates a spatial and temporal opportunity model and maps the opportunity to a two-dimensional coordinate system as shown in Fig. 2. Joint spatio-temporal sensing can be regarded as a binary decision between $\mathcal{O}_{1}$ (the first quadrant) and $\mathcal{O}_{0}$ (the second-third-fourth quadrants), where $\mathcal{O}_{0}$ denotes that a spatio-temporal opportunity is available and $\mathcal{O}_{1}$ represents otherwise.

\footnotetext{
${ }^{1}\lfloor x\rfloor$ corresponds an operation that rounds $x$ to the nearest integers less than or equal to $x$.
} 




Fig. 2. An illustrative graph for two-dimensional opportunity model. For a given cognitive sensor, an access opportunity exists $\left(\mathcal{O}_{0}\right)$ when the primary signal is temporally absent $\left(\mathcal{H}_{0}\right)$ or the sensor is outside the PER $\left(\mathcal{S}_{0}\right)$; no access opportunity exists $\left(\mathcal{O}_{1}\right)$ when the primary signal is temporally present $\left(\mathcal{H}_{1}\right)$ and the sensor is located inside the PER $\left(\mathcal{S}_{1}\right)$.

The pure temporal sensing can be considered as a binary decision between left plane and right plane (Fig. 2) and described as the following binary hypothesis testing problem [16]:

$$
\left\{\begin{array}{l}
\mathcal{H}_{0}: x_{i}[m]=w_{i}[m] \\
\mathcal{H}_{1}: x_{i}[m]=\sqrt{P_{i}\left(d_{i}\right)} \cdot s[m]+w_{i}[m],
\end{array}\right.
$$

where null hypothesis $\mathcal{H}_{0}$ represents the absence of the primary signal and hypothesis $\mathcal{H}_{1}$ denotes the presence of the primary signal. $x_{i}[m]$ is the received signal by cognitive sensor $i$ at time instant $m=1,2, \ldots, M$, where $M$ is the number of collected samples during one sampling process. $w_{i}[m] \sim \mathcal{N}\left(0, \sigma_{n, i}^{2}\right)$ is the additive white Gaussian noise sample, and $s[m]$ is the unattenuated sample (normalized to have unit power) of the primary transmit signal.

Similarly, the pure spatial sensing can be considered as a binary decision between upper plane and lower plane (Fig. 2) and modeled as another hypothesis testing problem [29]:

$$
\left\{\begin{array}{l}
\mathcal{S}_{0}: D_{p}<d_{i} \leq D_{s} \\
\mathcal{S}_{1}: 0 \leq d_{i} \leq D_{p},
\end{array}\right.
$$

where $\mathcal{S}_{0}$ denotes the case that cognitive sensor $i$ is located outside the PER and a spatial opportunity is available for it. $\mathcal{S}_{1}$ denotes the case that cognitive sensor $i$ is located inside the PER and no spatial opportunity can be exploited.

Therefore, from a two-dimensional detection perspective, we model the problem of spatio-temporal spectrum sensing for each cognitive sensor as a new hypothesis testing:

$$
\left\{\begin{array}{l}
\mathcal{O}_{0}: \mathcal{H}_{0} \cup \mathcal{S}_{0} \\
\mathcal{O}_{1}: \mathcal{H}_{1} \cap \mathcal{S}_{1}
\end{array}\right.
$$

where $\mathcal{O}_{0}$ denotes the case that a spatio-temporal opportunity is available, either because of the absence of the primary signal $\left(\mathcal{H}_{0}\right)$ or the cognitive sensor is located outside the PER $\left(\mathcal{S}_{0}\right)$. $\mathcal{O}_{1}$ represents the case that no spatio-temporal opportunity can be utilized, which means that the cognitive sensor is located

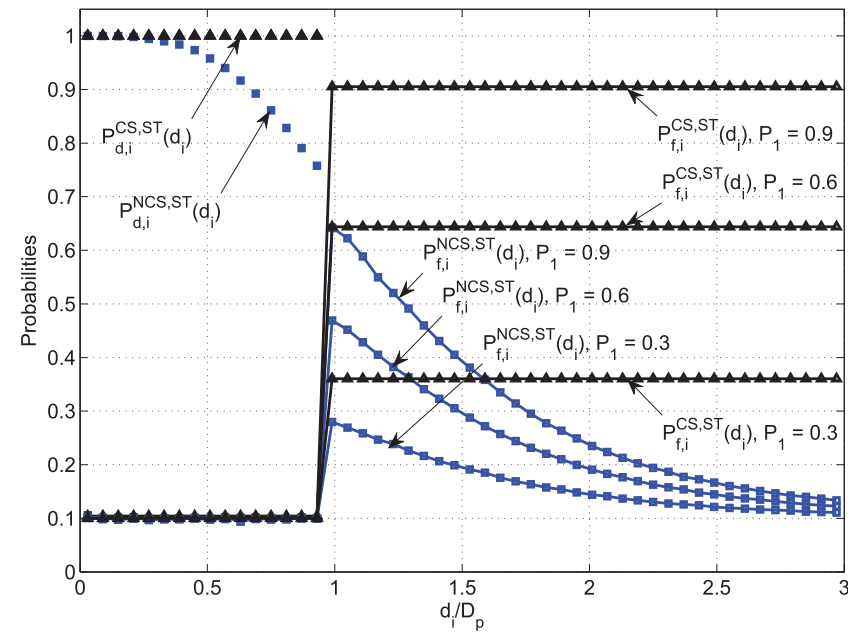

Fig. 3. Comparisons of spatio-Temporal detection performance between non-cooperative sensing (NCS) and OR-rule based cooperative sensing (CS) schemes. The detailed sensing parameter configuration is given in Section V.

inside the PER $\left(\mathcal{S}_{1}\right)$ and the PT is transmitting $\left(\mathcal{H}_{1}\right)$ at the same time.

Apparently, (4) is a straightforward combination of (2) and (3), while it is more general in opportunity-heterogeneous CSNs due to the integral consideration of primary user's traffic variations in both time and space domains. Moreover, it brings new technical challenges, e.g., the design of proper performance metrics and efficient sensing algorithms.

\section{Spatio-Temporal Detection Performance METRICS}

In this section, we introduce new detection performance metrics to guide the joint detection of spatio-temporal opportunities. The probabilities of spatio-temporal false alarm and detection for individual cognitive sensor $i$ are respectively given by

$$
\begin{aligned}
P_{f, i}^{S T}\left(d_{i}\right) & \triangleq \operatorname{Pr}\left\{\delta_{i}^{S T}\left(d_{i}\right)=\mathcal{O}_{1} \mid \mathcal{O}_{i}=\mathcal{O}_{0}\right\} \\
& =\left\{\begin{array}{l}
\operatorname{Pr}\left\{\delta_{i}^{S T}\left(d_{i}\right)=\mathcal{O}_{1} \mid \mathcal{H}_{0}\right\}, 0 \leq d_{i} \leq D_{p} \\
P_{1} \cdot \operatorname{Pr}\left\{\delta_{i}^{S T}\left(d_{i}\right)=\mathcal{O}_{1} \mid \mathcal{H}_{1}\right\} \\
+P_{0} \cdot \operatorname{Pr}\left\{\delta_{i}^{S T}\left(d_{i}\right)=\mathcal{O}_{1} \mid \mathcal{H}_{0}\right\}, D_{p}<d_{i} \leq D_{s}
\end{array}\right. \\
P_{d, i}^{S T}\left(d_{i}\right) & \triangleq \operatorname{Pr}\left\{\delta_{i}^{S T}\left(d_{i}\right)=\mathcal{O}_{1} \mid \mathcal{O}_{i}=\mathcal{O}_{1}\right\} \\
& =\operatorname{Pr}\left\{\delta_{i}^{S T}\left(d_{i}\right)=\mathcal{O}_{1} \mid \mathcal{H}_{1}\right\}, 0 \leq d_{i} \leq D_{p},
\end{aligned}
$$

where $\mathcal{O}_{i}$ is the actual hypothesis of cognitive sensor $i$ with a distance $d_{i}$ from the PT and $\delta_{i}^{S T}\left(d_{i}\right)$ denotes its binary decision result. $P_{1}\left(P_{0}\right)$ denotes the probability that the primary transmission is present (absent). Please refer to our prior works in [30] for detailed derivations of (5) and (6).

In terms of spatio-temporal detection performance metrics, Fig. 3 compares detection performance between between noncooperative sensing (NCS) and OR-rule based cooperative sensing (CS) schemes. It is shown in Fig. 3 that for a given temporal false alarm probability as 0.1 , when cognitive sensor $i$ is located inside the PER (i.e., $d_{i} \leq D_{p}$ ), CS scheme obtains a much higher detection probability than NCS scheme. However, when cognitive sensor $i$ is located outside the PER, 
CS scheme brings a much higher false alarm probability than NCS scheme. This is mainly due to the fact that, in CS scheme, the temporal detection probability of a cognitive sensor located far away from the PT (e.g, $d_{i}=2 D_{p}$ ) has been greatly improved, which actually results in severe spatial false alarms. Moreover, it is shown that the spatio-temporal false alarms are highly related to the primary activity level $P_{1}$, which is consistent with the analytical results in (5) and (6).

Neither NCS nor CS performs well in opportunityheterogeneous CSNs. In NCS scheme, the cognitive sensor inside the PER has a low spatio-temporal detection reliability due to the random channel fading, which cannot provide enough protection for the potential primary receivers. In CS scheme, however, the cognitive sensor outside the PER has a very high spatio-temporal false alarm probability due to the blind multiuser cooperation diversity, which provides overprotection for the potential primary receivers and results in severe access opportunity loss for the cognitive sensors. These drawbacks motivate us to design an effective spatio-temporal sensing framework.

\section{Three-Phase Spatio-Temporal Sensing}

\section{A. Challenges and Design Rationale}

In considering spatio-temporal sensing in opportunityheterogeneous CSNs, many critical challenges arise. First of all, joint optimization of spatial and temporal spectrum sensing is needed, to maximize the utilization of both spatial and temporal access opportunities for cognitive sensors, while satisfying the strict protection requirement from the primary users. Secondly, accurate location information of the primary transmitter is vital to mitigate spatial false alarms, while in practice it is unavailable for cognitive sensors due to the lack of cooperation from the primary transmitter. Furthermore, the random topology distribution of cognitive sensors and the opportunity-heterogeneity among them make it intractable to obtain a close-form optimal sensing scheme. However, there are some insightful observations as follows that can guide us to design suboptimal but effective sensing schemes:

- For a given time instant, the temporal access opportunity (i.e., the absence of the primary signal) is in common for all cognitive sensors and, a global cooperation among multiple cognitive sensors can significantly improve the temporal detection performance by exploiting multiuser diversity as compared with a non-cooperative approach.

- For a given topology distribution, the spatial access opportunities may vary for cognitive sensors located at different positions, which makes blind global cooperation unusable. However, there typically exist similar spatial opportunities among neighboring cognitive sensors, which will benefit the spatial opportunity detection.

\section{B. Algorithm Framework}

The observations above guide us in proposing a three-phase spatio-temporal sensing (TP-STS) framework, which consists of the following sequential phases:

Phase 1-Global Cooperation for Temporal Opportunity Detection: In this phase, the primary signal's presence $\left(\mathcal{H}_{1}\right)$ or

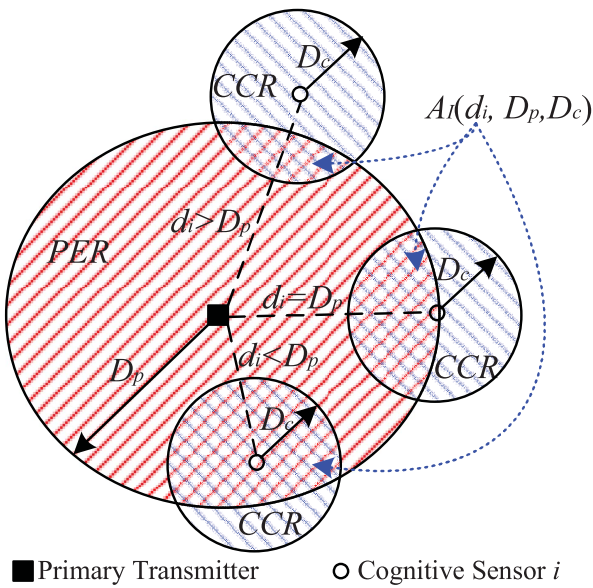

Fig. 4. Illustration of spatial opportunity detection. $D_{p}$ is the radius of primary exclusive region (PER), $D_{c}$ is the radius of cognitive cooperative region (CCR), and $d_{i}$ is the distance between the primary transmitter and cognitive sensor $i$. $A_{I}\left(d_{i}, D_{p}, D_{c}\right)$ denotes the common area of PER and CCR.

absence $\left(\mathcal{H}_{0}\right)$ is detected through cooperative sensing among all cognitive sensors. ${ }^{2}$ Here we use the classical OR logicbased decision fusion rule for its simplicity and superior performance over other schemes in temporal opportunity detection [33].

Specifically, in this phase a common receiver collects and fuses the binary decisions from each cognitive sensor and decides on the presence of a temporal access opportunity based on the following decision rule:

$$
T^{T}=\sum_{i=1}^{N} B_{i} \underset{\mathcal{H}_{0}}{\stackrel{\mathcal{H}_{1}}{\gtreqless}} 1,
$$

where $B_{i}$ is the non-cooperative binary decision of cognitive sensor $i$.

As shown in Fig. 3, for a given temporal false alarm probability, the temporal detection probability can be maintained at a very high level by using OR rule. On the other hand, the main drawback of the global cooperation is the severe spatial false alarm phenomena as also shown in Fig. 3, which will be processed in the following phase.

Phase 2-Local Cooperation for Spatial Opportunity Detection: Different from existing studies, this is a vital phase to tackle the spatial false alarms resulted from the global cooperation in Phase 1. In this phase, each cognitive sensor performs a binary hypothesis testing on whether it is located inside $\left(\mathcal{S}_{1}\right)$ or outside $\left(\mathcal{S}_{0}\right)$ the PER through effectively fusing the non-cooperative binary decisions of its one-hop neighbors.

As shown in Fig. 4, we consider the case that all one-hop neighbors of cognitive sensor $i$ are uniformly distributed in a disc area, named cognitive cooperative region (CCR), with cognitive sensor $i$ located at the center and a radius as $D_{c}$.

Specifically, denote $\mathbb{N}_{i}$ as the neighbor set of cognitive sensor $i$ and $\left|\mathbb{N}_{i}\right|=\left\lfloor\rho \pi D_{c}^{2}\right\rfloor$ as the total number of neighbors of cognitive sensor $i$. The decision rule at cognitive sensor $i$

\footnotetext{
${ }^{2}$ To further consider the issue of energy-efficiency in spectrum sensing, proper sensor selection and/or censoring schemes as those proposed in [35] and [36] could be integrated in this phase, which is not the focus of this paper.
} 
is given as:

$$
T_{i}^{S}=\sum_{k \in \mathbb{N}_{i}} B_{k} \underset{\mathcal{S}_{0}}{\stackrel{\mathcal{S}_{1}}{\gtrless}} \beta, \forall i=1, \ldots, N,
$$

where $\beta$ is the spatial detection threshold. It is noted that the decision rule in (8) is in essence a spatial $\beta$-out-of$\left|\mathbb{N}_{i}\right|$ rule. That is, if the number of its neighbors which make non-cooperative decisions as 'no opportunity' (i.e., $B_{k}=1, k \in \mathbb{N}_{i}$ ) is larger than $\beta$, cognitive sensor $i$ will declare itself as located inside the PER; otherwise, cognitive sensor $i$ will declare itself as located outside the PER.

Notably, the spatial detection threshold $\beta$ is a vital parameter to balance the tradeoff between the protection for the primary users and the spatial opportunity utilization of the cognitive sensors. If $\beta$ is much too small, more cognitive sensors located outside the PER $\left(\mathcal{S}_{0}\right)$ will potentially declare as inside $\left(\mathcal{S}_{1}\right)$, and if $\beta$ is much too large, more cognitive sensors located inside the PER $\left(\mathcal{S}_{0}\right)$ will potentially declare as outside $\left(\mathcal{S}_{0}\right)$. Generally, for any cognitive sensor $i$, the optimal spatial threshold can be obtained through solving the following optimization problems:

$$
\begin{array}{cc}
\text { OP1: } & \min _{\beta} \operatorname{Pr}\left\{T_{i}^{S} \geq \beta \mid S_{i}=S_{0}\right\} \\
& \text { Subject to } \operatorname{Pr}\left\{T_{i}^{S} \leq \beta \mid S_{i}=S_{1}\right\} \leq \bar{P}_{m d}^{S}
\end{array}
$$

or

$$
\text { OP2: } \begin{aligned}
\min _{\beta} & \left\{\operatorname{Pr}\left(S_{i}=S_{0}\right) \cdot \operatorname{Pr}\left\{T_{i}^{S} \geq \beta \mid S_{i}=S_{0}\right\}\right. \\
+ & \left.\operatorname{Pr}\left(S_{i}=S_{1}\right) \cdot \operatorname{Pr}\left\{T_{i}^{S} \leq \beta \mid S_{i}=S_{1}\right\}\right\},
\end{aligned}
$$

where OP1 and OP2 are respectively given according to the classical Neyman-Pearson criterion and Bayesian criterion in distributed detection problems [37]. $\bar{P}_{m d}^{S}$ in (9) is the maximum tolerant probability of spatial miss detection required by the primary users.

Due to the random topology distribution of cognitive sensors, in practice it is intractable to derive a close-form optimal solution to either (9) or (10). In this paper we propose two heuristic but effective spatial detection thresholds as follows

$$
\begin{gathered}
\beta_{1}=\left\lfloor\left|\mathbb{N}_{i}\right| \cdot \frac{A_{I}\left(d_{i}, D_{p}, D_{c}\right)}{\pi D_{c}^{2}}\right\rfloor_{d_{i}=D_{p}} \\
=\left\lfloor\frac { | \mathbb { N } _ { i } | } { \pi } \cdot \left\{\frac{D_{p}^{2}}{D_{c}^{2}} \cdot \arccos \left(1-\frac{D_{c}^{2}}{2 D_{p}^{2}}\right)+\arccos \left(\frac{D_{c}}{2 D_{p}}\right)\right.\right. \\
-\sqrt{\left.\left.\frac{D_{p}^{2}}{D_{c}^{2}}-\frac{1}{4}\right\}\right\rfloor,} \\
\beta_{2}=\left\lfloor\sum_{k \in \mathbb{N}_{i}^{\text {in }}} 1 \cdot \operatorname{Pr}\left\{B_{k}=1 \mid S_{k}=S_{1}\right\}\right. \\
\left.+\sum_{k \in \mathbb{N}_{i}^{\text {out }}} 1 \cdot \operatorname{Pr}\left\{B_{k}=1 \mid S_{k}=S_{0}\right\}\right\rfloor_{d_{i}=D_{p}} \\
\approx\left\lfloor\left|\mathbb{N}_{i}\right| \cdot \operatorname{Pr}\left\{B_{i}=1 \mid S_{i}=S_{1}\right\}\right\rfloor_{d_{i}=D_{p}}
\end{gathered}
$$

where $A_{I}\left(d_{i}, D_{p}, D_{c}\right)$ denotes the common area of PER and CCR (See Appendix B for general results on $A_{I}\left(d_{i}, D_{p}, D_{c}\right)$ ). $\mathbb{N}_{i}^{\text {in }}$ and $\mathbb{N}_{i}^{\text {out }}$ respectively denote the sets of cognitive sensor $i$ 's neighbors that are located inside and outside the PER.
For any cognitive sensor $i$ in the CSN, the design principles of the spatial thresholds $\beta_{1}$ and $\beta_{2}$ are summarized as follows:

- As shown in (3), the task of spatial opportunity detection in essence is to determine the relative relationship between $d_{i}$ and $D_{p}$, which means that the design of any proper spatial threshold should directly relate to $D_{p}$. Therefore, in designing both $\beta_{1}$ and $\beta_{2}$, we focus the study on the cognitive sensor located just at the edge of PER (i.e., $d_{i}=D_{p}$ ).

- As shown in Fig. 4 , the common area $A_{I}\left(d_{i}, D_{p}, D_{c}\right)$ of PER and CCR corresponds to the neighbors of cognitive sensor $i$ that are located inside the PER. In the case of uniform sensor distribution, $\beta_{1}$ represents the number of cognitive sensor $i$ 's neighbors that are located inside the PER. Intuitively, if cognitive sensor $i$ itself is located inside the PER, more than $\beta_{1}$ neighbors in the CCR will be located inside the PER, and vice versa.

- The spatial threshold $\beta_{2}$ is proposed to further integrate the impact of imperfect non-cooperative decisions. That is, a neighbor located inside the PER may declare $B_{k}=$ $0, k \in \mathbb{N}_{i}^{\text {in }}$; and a neighbor located outside the PER may declare $B_{k}=1, k \in \mathbb{N}_{i}^{\text {out }}$. The first term on the right side of Eq. (12) represents the average number of neighbors that are located inside the PER and correctly make local their decisions as 'inside the PER' and the second term represents the average number of the neighbors that are located outside the PER and falsely make local decisions as 'inside the PER'. In practice, due to the lack of the relative location information of the primary transmitter and its neighbors, cognitive sensor $i$ cannot obtain the non-cooperative detection performance of its neighbors, i.e., $\operatorname{Pr}\left\{B_{k}=1 \mid S_{k}=S_{1}\right\}$, for $k \in \mathbb{N}_{i}^{\text {in }}$ or $\operatorname{Pr}\left\{B_{k}=\right.$ $\left.1 \mid S_{k}=S_{0}\right\}$, for $k \in \mathbb{N}_{i}^{\text {out }}$. Consequently, an approximation is made by only using the local detection probability of the cognitive sensor $i$ located at the edge of the PER to obtain $\beta_{2}$. The effectiveness of this approximation will be demonstrated in the following section.

Phase 3-Joint Decision for spatio-temporal Opportunity: In this phase, a final decision on the availability of the spatiotemporal opportunity is obtained as follows:

$$
\left\{\begin{array}{l}
\text { If } T^{T}<1 \text { or } T_{i}^{S}<\beta, \text { then } \delta_{i}^{S T}\left(d_{i}\right)=\mathcal{O}_{0} \\
\text { If } T^{T}>1 \text { and } T_{i}^{S}>\beta, \text { then } \delta_{i}^{S T}\left(d_{i}\right)=\mathcal{O}_{1} .
\end{array}\right.
$$

\section{Numerical Results AND Discussions}

In this section, we present numerical simulations to demonstrate the effectiveness of the proposed TP-STS. The transmission power of the PT is assumed to be $25 \mathrm{~mW}$. The bandwidth $B$ is $200 \mathrm{KHz}$ and the sensing duration $T_{S}$ is $1 \mathrm{~ms}$. The receiver noise power spectral density is $-174 \mathrm{dBm} / \mathrm{Hz}$ and the receiver noise figure is $11 \mathrm{~dB}$. The path-loss exponent is 4 , the shadow fading $\mathrm{dB}$-spread is $5.5 \mathrm{~dB}$, and the average multipath Rayleigh fading gain is 1 . At the edge of the PER, the average received signal power is $-114 \mathrm{dBm}$, which amounts to a $D_{p}$ of $1.58 \mathrm{~km}$. The cognitive sensors are randomly located around the primary transmitter and the average sensor density is set to $\rho=40 / \mathrm{Km}^{2}$. The simulation results are 


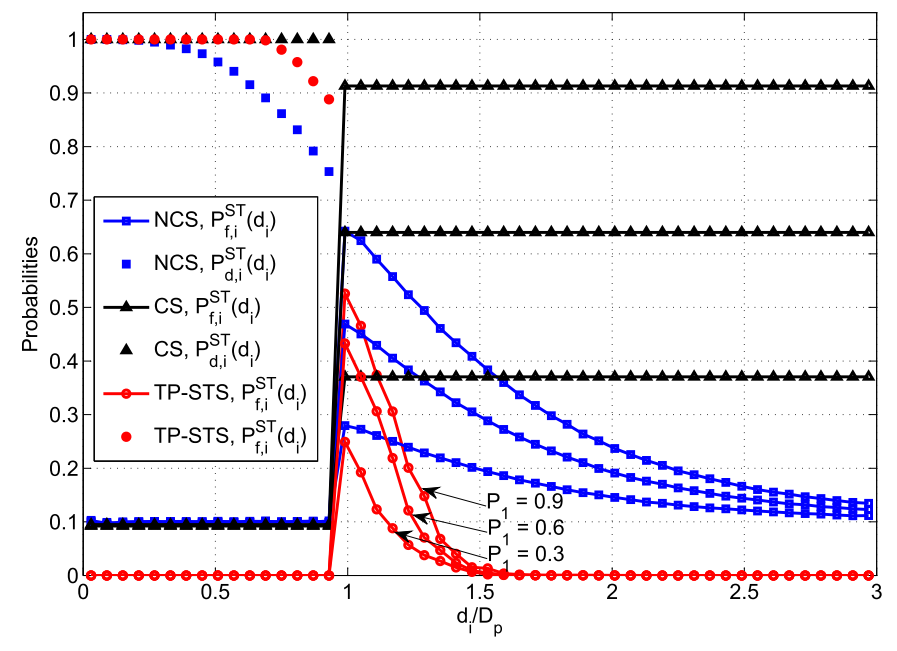

(a) $\beta=\beta_{1}$.

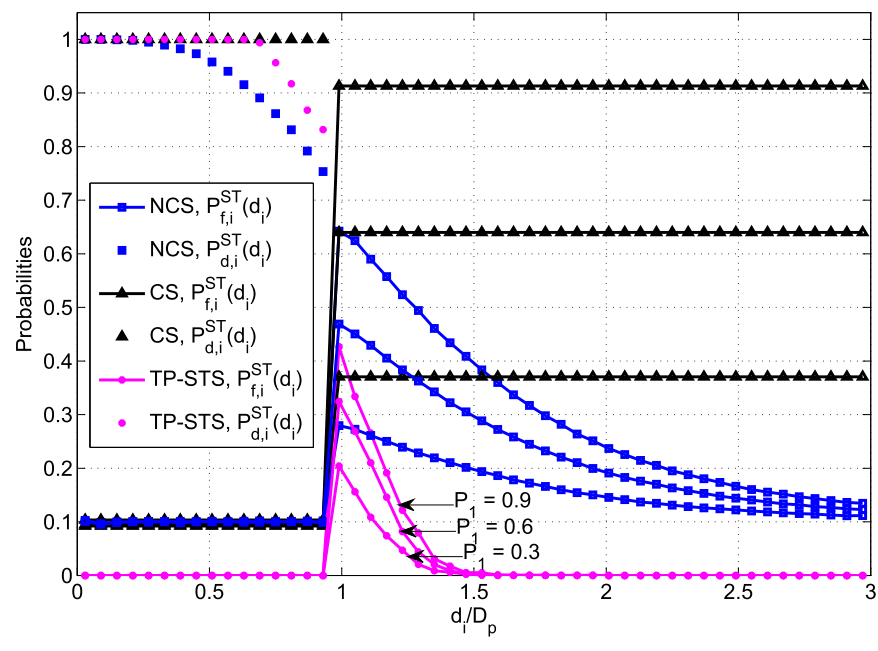

(b) $\beta=\beta_{2}$.

Fig. 5. Comparisons of the two-dimensional detection performance of NCS, CS and the proposed TP-STS $\left(D_{c}=D_{p} / 4\right)$. (a) For the proposed TP-STS, spatial threshold is set as $\beta=\beta_{1}$. (b) For the proposed TP-STS, spatial threshold is set as $\beta=\beta_{2}$.

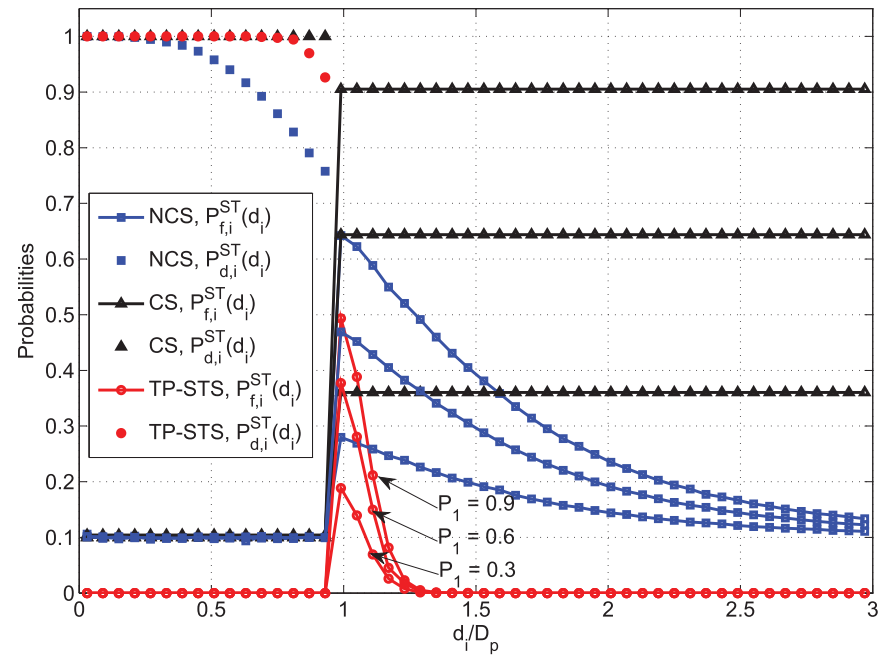

(a) $\beta=\beta_{1}$.

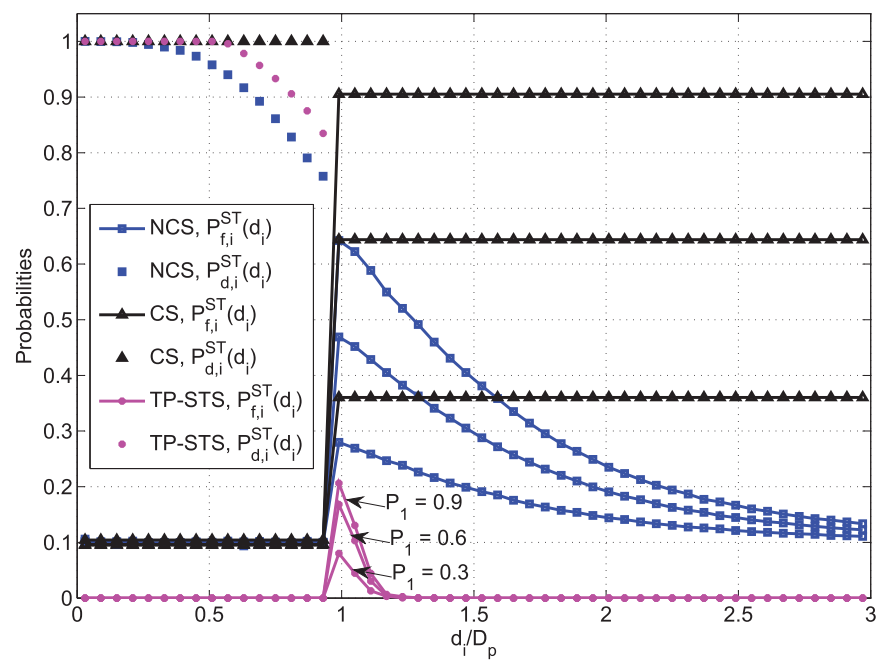

(b) $\beta=\beta_{2}$.

Fig. 6. Comparisons of the two-dimensional detection performance of NCS, CS and the proposed TP-STS $\left(D_{c}=D_{p} / 2\right)$. (a) For the proposed TP-STS, spatial threshold is set as $\beta=\beta_{1}$. (b) For the proposed TP-STS, spatial threshold is set as $\beta=\beta_{2}$.

obtained by averaging the results of 100 randomly-generated topologies and $10^{4}$ random channel realizations. To make a fair comparison, the temporal false alarm probabilities for all schemes are set as 0.1 .

Fig. 5 and Fig. 6 presents the comparison of NCS scheme, CS scheme and the proposed TP-STS scheme, in terms of the spatio-temporal detection performance. It is shown in both Fig. 5 and Fig. 6 that: (i) The spatio-temporal false alarm probability of the proposed TP-STS scheme declines to almost zero when $d_{i} / D p<1$ and $d_{i} / D p>2$, which greatly decreases the amount of access opportunity loss and improves the spectrum utilization for cognitive sensors. The reason behind is that in TP-STS scheme, most of the potential false alarms resulted from the global cooperation phase can be corrected in the sequential local cooperation phase, here we call this performance gain as multi-phase diversity gain. (ii) The spatio-temporal detection probability of the proposed TP-STS scheme, using either $\beta_{1}$ or $\beta_{2}$, is worse than CS scheme but significantly better than NCS scheme. Specifically, using $\beta_{1}$ provides a higher detection probability and thus more protection for the primary users, while using $\beta_{2}$ results in a smaller spatio-temporal false alarm probability and thus higher spectrum utilization for cognitive sensors.

By comparing the results in Fig. 5 and Fig. 6, it is observed that the range of CCR, i.e., $D_{c}$, has an important impact on the detection performance of the proposed TP-STS. Apparently, when the distance between the cognitive sensor $i$ and the PT satisfies $d_{i} / D_{p} \in[1,1.5]$, the spatio-temporal false alarm probabilities of the proposed TP-STS scheme in Fig. 6 with $D_{c}=D_{p} / 2$ are smaller than those in Fig. 5 with $D_{c}=D_{p} / 4$.

\section{RELATED WORK}

The subject of opportunity-heterogeneity among different cognitive sensors has been studied in the existing body of research on dynamic spectrum access strategies in terms of 
overlay and underlay [9]. In overlay strategy, cognitive sensors can only exploit the temporal opportunity in the absence of the primary transmission. However, in underlay strategy, cognitive sensors can always access the licensed spectrum subject to an interference temperature constraint from the primary use. Mixed access strategy of overlay and underlay has also been recently studied to exploit temporal and spatial opportunities jointly [21]. The problem of cooperative sensing among opportunity-heterogeneous cognitive sensors has been first formulated in [22] and an extension to wideband sensing has been presented in [23]. Differently, in this paper we investigate the issue of spectrum sensing in opportunityheterogeneous CSNs from a perspective of joint exploration of spatio-temporal opportunities, which remains a significant challenge due to the requirement of integrating the spatial and temporal information with spectrum sensing.

The topic of designing proper performance metrics to evaluate the effectiveness of spectrum sensing algorithms has also received growing attention. As mentioned in Section I, traditionally, a probability of detection (i.e., the probability of correctly determining that the primary transmission is present) as a function of a probability of false alarm (i.e., the probability of incorrectly determining that the primary transmission is present) is used for temporal sensing [9]. In [24], the issue of spatial false alarm problem involved in the pure temporal performance metrics is highlighted to characterize the access opportunity loss in spatial domain. In [25], the authors introduce space-time detection metrics to characterize the tradeoff between the safety to primary users and the spectrum utilization of a single cognitive sensor, which may be located either inside or outside a primary exclusive region (PER) (see Fig.1). The safety is measured in terms of the harmful interference caused by a cognitive sensor located at the worst-case location, i.e., the edge of the PER. The spectrum utilization is measured by a weighted (with respect to all possible locations of the cognitive sensor) probability of spectrum holes recovered by the cognitive sensor. Differently, the unique features of the presented metrics in this paper are two-folds. The first is that the presented metrics can characterize the spatio-temporal detection performance of cognitive sensors under a more general location assumption. The second is that the presented metrics can effectively evaluate both NCS algorithms and CS algorithms, while the metrics in [25] are not yet easily to be extended to multi-sensor CS algorithms. More importantly, the presented metrics can be used to guide the design of efficient sensing algorithms for opportunityheterogeneous CSNs, which are not considered in [25].

\section{CONCLUSION}

The spectrum access opportunities for cognitive sensors exist not only in time domain but also in space domain. In this paper we introduced a novel opportunity-heterogeneous cognitive sensor network (CSN) model and redefined the problem of spectrum sensing in opportunity-heterogeneous CSNs from a joint spatio-temporal two-dimensional detection perspective. We showed the limitations and original drawbacks of pure temporal-domain detection performance metrics, which motivated us to derive novel spatio-temporal detection performance metrics and to further propose an efficient threephase spatio-temporal sensing (TP-STS) algorithm to tackle the problem of spectrum sensing in opportunity-heterogeneous CSNs by exploiting the multi-phase diversity gain. Numerical results were also provided to demonstrate the effectiveness of the proposed algorithm.

\section{APPENDIX A}

\section{Calculation of Common ARea of PER and CCR}

As shown in Fig. $5, D_{p}$ and $D_{c}$ respectively denote the radius of PER and CCR, $d_{i}$ denotes the distance between the primary transmitter and cognitive sensor $i$, the intersecting area of PER and CCR can be calculated as follows:

Case I: for $0 \leq d_{i} \leq\left|D_{p}-D_{c}\right|$,

$$
A_{I}\left(d_{i}, D_{p}, D_{c}\right)=\pi \min \left\{D_{p}^{2}, D_{c}^{2}\right\}
$$

Case II: for $\left|D_{p}-D_{c}\right|<d_{i}<D_{p}+D_{c}$,

$$
\begin{aligned}
A_{I}\left(d_{i}, D_{p}, D_{c}\right)= & D_{p}^{2} \arccos \left(\frac{D_{p}^{2}+d_{i}^{2}-D_{c}^{2}}{2 D_{p} d_{i}}\right) \\
& +D_{c}^{2} \arccos \left(\frac{D_{c}^{2}+d_{i}^{2}-D_{p}^{2}}{2 D_{c} d_{i}}\right) \\
& -d_{i} \sqrt{D_{c}^{2}-\left(\frac{D_{c}^{2}+d_{i}^{2}-D_{p}^{2}}{2 d_{i}}\right)^{2}} .
\end{aligned}
$$

Case III: for $d_{i} \geq D_{p}+D_{c}, A_{I}\left(d_{i}, D_{p}, D_{c}\right)=0$.

\section{ACKNOWLEDGMENT}

The authors would like to thank the anonymous reviewers and the Associate Editor for their constructive comments and Dr. Xian Zhang and Dr. Zongsheng Zhang for their valuable input.

\section{REFERENCES}

[1] D. Puccinelli and M. Haenggi, "Wireless sensor networks: Applications and challenges of ubiquitous sensing," IEEE Circuits Syst. Mag., vol. 5, no. 3, pp. 19-31, Sep. 2005.

[2] G. Zhou, J. A. Stankovic, and S. H. Son, "Crowded spectrum in wireless sensor networks," in Proc. EmNets, May 2006, pp. 1-6.

[3] S. Haykin, "Cognitive radio: Brain-empowered wireless communications," IEEE J. Sel. Areas Commun., vol. 23, no. 2, pp. 201-220, Feb. 2005

[4] O. Akan, O. Karli, and O. Ergul, "Cognitive radio sensor networks," IEEE Netw., vol. 23, no. 4, pp. 34-40, Jul. 2009.

[5] H. G. Goh, K. H. Kwong, C. Shen, C. Michie, and I. Andonovic, "CogSeNet: A concept of cognitive wireless sensor network," in Proc. 7th IEEE Consum. Commun. Netw. Conf., Jan. 2010, pp. 1-2.

[6] A. S. Zahmati, S. Hussain, X. Fernando, and A. Grami, "Cognitive wireless sensor networks: Emerging topics and recent challenges," in Proc. IEEE Toronto Int. Conf. Sci. Technol. Humanity, Sep. 2009, pp. 593-596.

[7] G. Vijay, E. Bdira, and M. Ibnkahla, "Cognition in wireless sensor networks: A perspective," IEEE Sensors J., vol. 11, no. 3, pp. 582-592, Mar. 2011.

[8] R. Tandra, S. M. Mishra, and A. Sahai, "What is a spectrum hole and what does it take to recognize one?" Proc. IEEE, vol. 97, no. 5, pp. 824-848, May 2009.

[9] Q. Zhao and B. M. Sadler, "A survey of dynamic spectrum access: Signal processing, networking, and regulatory policy," IEEE Signal Process. Mag., vol. 24, no. 3, pp. 79-89, May 2007.

[10] D. Cabric, S. M. Mishra, and R. Brodersen, "Implementation issues in spectrum sensing for cognitive radios," in Proc. 38rd Asilomar Conf. Signals, Syst., Comput., Nov. 2004, pp. 772-776. 
[11] T. Yucek and H. Arslan, "A survey of spectrum sensing algorithms for cognitive radio applications," IEEE Commun. Surv. Tuts., vol. 11, no. 1, pp. 116-130, Mar. 2009.

[12] Y. Zeng, Y.-C. Liang, A. T. Hoang, and R. Zhang, "A Review on spectrum sensing for cognitive radio: Challenges and solutions," EURASIP J. Adv. Signal Process. vol. 2010, pp. 381465-1-381465-15, Jan. 2010.

[13] I. F. Akyildiz, B. F. Lo, and R. Balakrishnan, "Cooperative spectrum sensing in cognitive radio networks: A survey," Phys. Commun., vol. 4, no. 1, pp. 40-62, Mar. 2011.

[14] D. Duan, L. Yang, and J. C. Principe, "Cooperative diversity of spectrum sensing for cognitive radio systems," IEEE Trans. Signal. Process. vol. 58, no. 6, pp. 3218-3227, Jun. 2009.

[15] E. Visotsky, S. Kuffner, and R. Peterson, "On collaborative detection of TV transmissions in support of dynamic spetrum sharing," in Proc. 1st IEEE Int. Symp. IEEE New Frontiers Dyn. Spectr. Access Netw. Nov. 2005, pp. 338-345.

[16] J. Ma, G. Zhao, and Y. Li, "Soft combination and detection for cooperative spectrum sensing in cognitive radio networks," IEEE Trans. Wireless Commun., vol. 7, no. 11, pp. 4502-4507, Nov. 2008

[17] E. Peh, Y.-C. Liang, Y. L. Guan, and Y. Zeng, "Optimization of cooperative sensing in cognitive radio networks: A sensing-throughput tradeoff view," IEEE Trans. Veh. Tech., vol. 58, no. 9, pp. 5294-5299, Nov. 2009.

[18] Q. Wu, G. Ding, J. Wang, X. Li, and Y. Huang, "Consensus-based decentralized clustering for cooperative spectrum sensing in cognitive radio networks," Chin. Sci. Bull., vol. 57, nos. 28-29, pp. 3677-3683, Oct. 2012.

[19] G. Ding, Q. Wu, Y. D. Yao, J. Wang, and Y. Chen, "Kernelbased learning for statistical signal processing in cognitive radio networks," IEEE Signal Process. Mag., vol. 30, Jul. 2013, DOI: 10.1109/MSP.2013.2251071.

[20] IEEE Standard for Wireless Regional Area Networks Part 22: Cognitive Wireless RAN Medium Access Control (MAC) and Physical Layer (PHY) Specifications: Policies and Procedures for Operation in the TV Bands, IEEE Standard 802.22-2011, Jul. 2011.

[21] M. G. Khoshkholgh, K. Navaie, and H. Yanikomeroglu, "Access strategies for spectrum sharing in fading environment: Overlay, underlay and mixed," IEEE Trans. Mobile Comput., vol. 9, no. 12, pp. 1780-1793, Dec. 2010

[22] H. Li, "Cooperative spectrum sensing via belief propagation in spectrumheterogeneous cognitive radio systems," in Proc. IEEE Wireless Commun. Netw. Conf., Apr. 2010, pp. 1-6.

[23] Z. Zhang, Z. Han, H. Li, and D. Yang, "Belief-propagation based cooperative spectrum sensing in wideband cognitive radio networks," IEEE Trans. Wireless Commun., vol. 10, no. 9, pp. 3020-3031, Sep. 2011.

[24] W. Han, J. Li, Q. Liu, and L. Zhao, "Spatial false alarms in cognitive radio," IEEE Commun. Lett., vol. 15, no. 5, pp. 518-520, May 2011.

[25] R. Tandra, A. Sahai, and V. V. Veeravalli, "Space-time metrics for spectrum sensing," in Proc. IEEE Symp. New Frontiers Dyn. Spectr. Apr. 2010, pp. 1-12.

[26] M. Vu, N. Devroye, and V. Tarokh, "On the primary exclusive region of cognitive networks," IEEE Trans. Wireless Commun., vol. 8, no. 7, pp. 3380-3385, Jul. 2009.

[27] J. Ma, G. Li, and B. H. Juang, "Signal processing in cognitive radio," Proc. IEEE, vol. 97, no. 5, pp. 805-823, May 2009.

[28] A. Goldsmith, Wireless Communications. Cambridge, U.K.: Cambridge Univ. Press, 2005.

[29] S. M. Mishra, "Maximizing available spectrum for cognitive radios," Ph.D. dissertation, EECS Dept., Univ. of California, Berkeley, Oakland, CA, USA, Jan. 2010

[30] Q. Wu, G. Ding, J. Wang, and Y.-D. Yao, "Spatial-temporal opportunity detection in spectrum-heterogeneous cognitive radio networks: Twodimensonial sensing," IEEE Trans. Wireless Commun., vol. 12, no. 2, pp. 516-526, Feb. 2013.

[31] W. Zhang and K. B. Letaief, "Cooperative spectrum sensing with transmit and relay diversity in cognitive networks," IEEE Trans. Wireless Commun., vol. 7, no. 12, pp. 4761-4766, Dec. 2008.

[32] W. Saad, Z. Han, T. Basar, M. Debbah, and A. Hjørungnes, "Coalition formation games for collaborative spectrum sensing," IEEE Trans. Vel. Tech., vol. 60, no. 1, pp. 276-297, Jan. 2011.

[33] A. Ghasemi and E. S. Sousa, "Opportunistic spectrum access in fading channels through collaborative sensing," J. Commun., vol. 2, no. 2, pp. 71-82, Mar. 2007.

[34] K. B. Letaief and W. Zhang, "Cooperative communications for cognitive radio networks," Proc. IEEE, vol. 97, no. 5, pp. 878-893, May 2009.
[35] S. Maleki, A. Pandharipande, and G. Leus, "Energy-efficient distributed spectrum sensing for cognitive sensor networks," IEEE Sensors J., vol. 11, no. 3, pp. 565-573, Mar. 2011.

[36] G. Ding, Q. Wu, F. Song, and J. Wang, "Decentralized sensor selection for cooperative spectrum sensing using unsuperivised learning," in Proc. 2012 IEEE Int. Conf. Commun. (ICC), Jun. 2012, pp. 1-6.

[37] P. K. Varshney, Distributed Detection and Data Fusion. New York, NY, USA: Springer-Verlag, 1997.

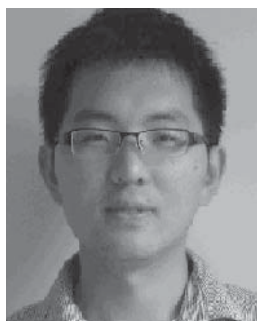

Guoru Ding (S'10) received the B.S. degree (Hons.) in electrical engineering from Xidian University, Xi' an, China, in 2008, and the M.S. degree from the Institute of Communications Engineering, PLA University of Science and Technology, Nanjing, China, in 2011 . He is currently pursuing the Ph.D. degree in communications and information systems with the College of Communications Engineering, PLA University of Science and Technology.

His current research interests include wireless sensor networks, cognitive radio networks, spatiotemporal signal processing, distributed optimization theory, and statistical learning. He currently serves as a Reviewer for the IEEE SIGNAL PROCESSing Magazine, the IEEE TRansactions on Communications, the IEEE TRANSACTIONS ON WIRELESS COMMUNICATIONS, and the IEEE TRANSACTIONS ON Vehicular TEChNOLOGY. He was a recipient of the Best Paper Awards from the IEEE WCSP in 2009. He is currently a voting member of IEEE 1900.7 White Space Radio Working Group.

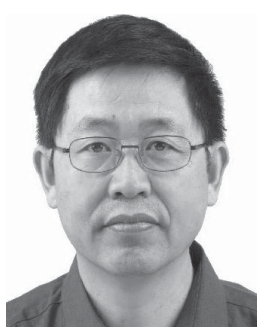

Jinlong Wang (SM'13) received the B.S. degree in wireless communications, and the M.S. and Ph.D. degrees in communications and electronic systems from the Institute of Communications Engineering, Nanjing, China, in 1983, 1986, and 1992, respectively.

$\mathrm{He}$ is currently a Chair Professor with the PLA University of Science and Technology, Nanjing. He is the Co-Chair of IEEE Nanjing Section. He has published widely in the areas of signal processing for wireless communications and networking. His current research interests include soft defined radio, cognitive radio, and green wireless communication systems.

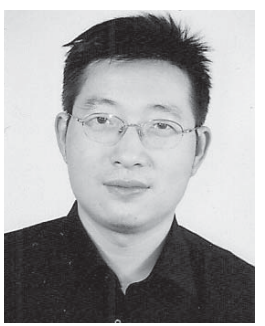

Qihui Wu (SM'12) received the B.S. degree in communications engineering, and the M.S. and Ph.D. degrees in communications and information systems from the Institute of Communications Engineering, Nanjing, China, in 1994, 1997, and 2000, respectively. From 2003 to 2005, he was a Postdoctoral Research Associate with Southeast University, Nanjing. From 2005 to 2007, he was an Associate Professor with the Institute of Communications Engineering, PLA University of Science and Technology, Nanjing, where he is currently a Full Professor. From March 2011 to September 2011, he was an Advanced Visiting Scholar with the Stevens Institute of Technology, Hoboken, NJ, USA.

His current research interests include wireless communications and statistical signal processing, with emphasis on system design of software defined radio, cognitive radio, and smart radio.

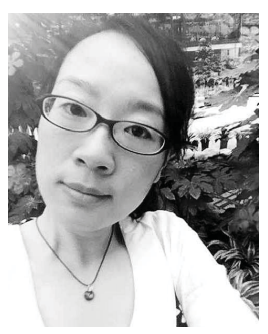

Fei Song received the B.S. degree in communications engineering and the Ph.D. degree in communications and information system from the Institute of Communications Engineering, PLA University of Science and Technology, Nanjing, China, in 2002 and 2007, respectively. She is currently a Lecturer with the PLA University of Science and Technology. Her current research interests include cognitive radio networks, MIMO, and statistical signal processing. 


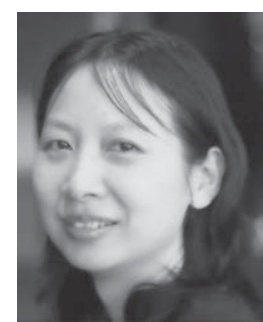

Yingying Chen (SM'11) is an Associate Professor with the Department of Electrical and Computer Engineering, Stevens Institute of Technology, Hoboken, NJ, USA. Prior to joining Stevens Institute of Technology, she was with Alcatel-Lucent, Murray Hill, NJ, USA. Her work has involved a combination of research and development of new technologies and real systems. She was an Instructor with the Computer Science Department, Rutgers University. She was a recipient of the NSF CAREER Award in 2010 and Google Research Award in 2010. She received the Stevens Board of Trustees Award for Scholarly Excellence in 2010. She is a recipient of the Best Paper Awards from ACM MobiCom in 2011 and WONS in 2009, as well as the Best Technological Innovation Award from the International TinyOS Technology Exchange in 2006. She received the IEEE Outstanding Contribution Award from the IEEE New Jersey Coast Section from 2005 to 2009.

She received the Ph.D. degree in computer science from Rutgers University, the M.S. degree in computer science from North Carolina State University, and the B.S. degree in physics from Nanjing University, Nanjing, China Her current research interests include cyber security and privacy, wireless embedded systems, wireless and sensor networks and mobility, mobile social networks, and pervasive computing. She has co-authored Securing Emerging Wireless Systems and he has published extensively in journal and conference papers. Her research has been reported in numerous media outlets, including the Wall Street Journal, MIT Technology Review, Inside Science, NPR, and CNET. 